Baltic Astronomy, vol. 9, 485-498, 2000.

\title{
WHITE DWARF DATA TABLES
}

\author{
P. A. Bradley
}

XTA, MS B220, Los Alamos National Laboratory, Los Alamos, NM 87545, U.S.A.

Received November 1, 1999

\begin{abstract}
The following tables give the updated photometric and physical parameters of variable white dwarfs together with lists of the representative references.
\end{abstract}

Key words: catalogs - stars: white dwarfs, oscillations, interacting binaries

\section{INTRODUCTION AND EXPLANATION}

Below a brief explanation of the information in the tables is given. In all cases, I list the WD number, either from the catalogue of McCook \& Sion (1999) or determined by me from the epoch 1950 coordinates. Next, I list the most commonly used name (or alias), then I list the variable star designation if it is available. If not, I list the constellation name and a $\mathrm{V}^{* *}$ or ?? depending on what the last designated variable star for that constellation is. I present epoch 2000 coordinates for all of the stars, which I precessed from the 1950 ones in most cases. I do not include proper motion effects; this is negligible for all except the largest proper motion DAV stars, such as L 19-2, BPM 37093, R 808 and G 29-38. Even in these cases, the error is no more than $30^{\prime \prime}$ in declination and $2 \mathrm{~s}$ in right ascension. I culled effective temperatures from the latest work (listed under each table); they are now much more homogeneous than before. I pulled the magnitude estimates from the appropriate paper, and they are mean values integrated over several cycles. The amplitude given is for the height of a typical pulse in the light curve. The periods correspond the dominant ones found in the light curve. In some 
cases, there is a band of power in a given period range, or the light curve is very complex, and I indicate this in the table.

In the references, I generally list the paper with the most comprehensive pulsation analysis for the star in question. In some cases, there is more than one good reference, and I list them as well. If you need more information on a particular star, I suggest consulting the reference list contained in the papers I list, especially for older references.

Finally, these tables are intended to be both useful and up to date. If you have corrections, suggestions for improvement, or updates, please contact me at bradley@bullwinkle.as.utexas.edu or pbradley@lanl.gov.

\section{Representative references for Table 1 (DAV stars)}

1. BPM 30551: Hesser J.E., Lasker B. M., Neupert H.E. 1976, ApJ, 209, 853; McGraw J. T. 1977, ApJ, 214, L123.

2. R 548: Lasker B. M., Hesser J. E. 1971, ApJ, 163, L89; Stover R. J., Hesser J.E., Lasker B. M., Nather R. E., Robinson E. R. 1980, ApJ, 240, 865; Kepler S. O. et al. 1995, in The Third WET Workshop Proceedings, eds. E. G. Meištas \& J.-E. Solheim, Baltic Astronomy, 4, 238.

3. BPM 31594: McGraw J. T. 1976, ApJ, 210, L35; O'Donoghue D. 1986, MNRAS, 220, 19P; O'Donoghue D., Warner B., Cropper M. 1992, MNRAS, 258, 415.

4. HL Tau 76: Page C. G. 1972, MNRAS, 159, 25P; Fitch W.S. 1973, ApJ, 181, L95; Dolez N., Kleinman S. J. 1997, in White Dwarfs, eds. J. Isern, M. Hernanz \& E. García-Berro, Kluwer, Dordrecht, p. 437.

5. G 38-29: McGraw J. T., Robinson E. L. 1975, ApJ, 200, L89; Kleinman S.J. 1995, Ph.D. Thesis, Univ. Texas.

6. G 191-16: McGraw J. T., Fontaine G., Dearborn D. S. P., Gustafson J., Lacombe P., Starrfield S. G. 1981, ApJ, 250, 349; Vauclair G. et al. 1991, A\&A, 215, L17; Kleinman S. J. 1995, Ph.D. Thesis, Univ. Texas.

7. HS0507+0435: Jordan S. et al. 1998, A\&A, 330, 227.

8. GD 66: Dolez N., Vauclair G., Chevreton M. 1983, A\&A, 121, L23; Fontaine G., Wesemael F., Bergeron P., Lacombe P., Lamontagne R., Saumon D. 1985, ApJ, 294, 339.

9. KUV 08368+4026: Vauclair G. et al. 1996, A\&A, 322, 155.

10. GD 99: McGraw J. T., Robinson E. L. 1976, ApJ, 205, L155. 
11. G 117-B15A: Kepler S. O., Robinson E. L., Nather R. E., McGraw J. T. 1982, ApJ, 254, 676; Kepler S. O. et al. 1991, ApJ, 378, L45; Kepler S. O. et al. 1995, in The Third WET Workshop Proceedings, eds. E. G. Meištas \& J.-E.Solheim, Baltic Astronomy, 4, 221.

12. KUV 11370+4222: Vauclair G. et al. 1996, A\&A, 322, 155.

13. G 255-2: Vauclair G., Dolez N., Chevreton M. 1983, A\&A, 103, L17.

14. BPM 37093: Kanaan A. N. et al. 1992, ApJ, 390, L89.

15. GD 154: Robinson E. L. Stover R. J., Nather R. E., McGraw J. T. 1978, ApJ, 220, 614; Pfeiffer B. et al. 1996, A\&A, 314, 182.

16. G 238-53: Fontaine G., Wesemael F. 1984, AJ, 89, 1728.

17. EC 14012-1446: Stobie R. S. et al. 1995, MNRAS, 272, L21.

18. GD 165: Becklin E. E., Zuckerman B. 1988, Nature, 336, 656. Bergeron P., McGraw J. T. 1990, ApJ, 352, L45; Bergeron P. et al. 1993, AJ, 106, 1987

19. L 19-2: O'Donoghue D., Warner B. 1982, MNRAS, 200, 573; O'Donoghue D., Warner B. 1987, MNRAS, 228, 949; Sullivan D. J. 1995, in The Third WET Workshop Proceedings, eds. E. G. Meištas \& J.-E. Solheim, Baltic Astronomy, 4, 261.

20. PG 1541+650: Vauclair G. et al. 1999, in Massive Photometry, (IAU Colloq. 176), ed. G. Kovács (ASP, San Francisco); Homeier et al. 1998, A\&A, 338, 563.

21. R 808: McGraw J. T., Robinson E. L. 1976, ApJ, 205, L155.

22. G 226-29: Kepler S. O., Robinson E. R., Nather R. E. 1983, ApJ, 271, 744; Kepler S.O. et al. 1995, ApJ, 447, 874.

23. BPM 24754: Giovannini O. et al. 1997, A\&A, 329, L13.

24. G 207-9: Robinson E. L., McGraw J. T. 1976, ApJ, 207, L37.

25. G 185-32: McGraw J. T., Fontiane G., Dearborn D. S. P., Gustafson J., Lacombe P., Starrfield S. G. 1981, ApJ, 250, 349.

26. GD 385: Kepler S. O. 1984, ApJ, 278, 754; O’Donoghue D. 1986, MNRAS, 220, 19P.

27. PG 2303+242: Vauclair G., Chevreton M., Dolez N. 1987, A\&A, 175, L13; Vauclair G. et al. 1992, A\&A, 264, 547.

28. G 29-38: McGraw J. T., Robinson E. L. 1975, ApJ, 200, L89; Winget D. E. et al. 1990, ApJ, 357, 630; Patterson J. et al. 1991, ApJ, 374, 330; Kleinman S. J. 1997, in White Dwarfs, eds. J. Isern, M. Hernanz \& E. García-Berro, Kluwer, Dordrecht, p. 459.

29. EC 23487-2424: Stobie R. S., Chen A., O'Donoghue D., Kilkenny D. 1993, MNRAS, 263, L13. 


\section{References for Table 2 (DAV star temperatures)}

[Ber] Bergeron P., Wesemael F., Lamontagne R., Fontaine G., Saffer R. A., Allard N.F. 1995, ApJ, 449, 258. Our values are from their Table 4.

[KA] Koester D., Allard N. 1993, White Dwarfs: Advances in Observations and Theory, ed. M. A. Barstow, Kluwer, Dordrecht, p. 237. For G 117-B15A, see: Koester D., Allard N.F., Vauclair G. 1994, A\&A, 291, L9.

[KN] Kepler S. O., Nelan E. P. 1993, AJ, 105, 608.

[Daou] Daou D., Wesemael F., Bergeron P., Fontaine G., Holberg J.B. 1990, ApJ, 364, 242. For G 226-29, see: Fontaine G., Brassard P., Bergeron P., Wesemael F. 1992, ApJ, 399, L91. For GD 165, see: Bergeron P. et. al. 1993, AJ, 106, 1987 For BPM 37093, see: Bragaglia A., Renzini A., Bergeron P. 1993, White Dwarfs: Advances in Observations and Theory, ed. M. A. Barstow, Kluwer, Dordrecht, p. 325.

[W86] Wesemael F., Lamontagne R., Fontaine G. 1986, AJ, 91, 1376. The effective temperature for GD 66 comes from Lamontagne R., Wesemael F., Fontaine G. 1987, The Second Conference on Faint Blue Stars, ed. A. G. D. Philip, D. S. Hayes \& J. Liebert, L. Davis Press, Schenectady, N.Y., p. 677.

[WK84] Weidemann V., Koester D. 1984, A\&A, 132, 195. The values for G 255-2 and G 207-9 come from Koester D., Schulz H., Weidemann V. 1979, A\&A, 76, 262. The values for BPM 30551, BPM 31594 and PG 2303+243 are my estimates based on model atmospheres of Koester (priv. comm.) and published Strömgren photometry of Fontaine et al. 1985, AJ, 90, 1094.

\section{Representative references for Table 3 (DBV stars)}

1. KUV 05134+2605: Grauer A.D., Wegner G., Liebert J. 1989, AJ, 98, 2221.

2. CBS 114: Winget D. E., Claver C. F. 1989, in White Dwarfs (IAU Colloq. 114), ed. G. Wegner, Springer, Berlin, p. 290.

3. PG 1115+158: Winget D. E., Nather R. E., Hill J. A. 1987, ApJ, 316, 305; Clemens J.C. et al. 1993, in White Dwarfs: Advances in Observation and Theory, ed. M. A. Barstow, Kluwer, Dordrecht, p. 515.

4. PG 1351+489: Winget D.E., Nather R. E., Hill J. A. 1987, ApJ, $316,305$. 
5. PG 1456+103: Grauer A. D., Bond H. E., Green R.F., Liebert J. 1988, AJ, 95, 879.

6. GD 358: Winget D. E., Robinson E. R., Nather R. E., Fontaine G. 1982, ApJ, 262, L11; Winget D. E. et al. 1994, ApJ, 430, 839; Bradley P. A., Winget D. E. 1994, ApJ, 430, 850; Provencal J. L. et al. 1996, ApJ, 466, 1011.

7. PG 1654+160: Winget D. E., Robinson E. L., Nather R. E., Balachandran S. 1984, ApJ, 279, L15.

8. EC 20058-5234: O'Donoghue D. 1995, 9th European Workshop on White Dwarf Stars, eds. D. Koester \& K. Werner, Springer, Berlin, p. 297.

\section{References for Table 4 (DBV star temperatures)}

[B:] Temperatures from Beauchamp A. et al. 1999, ApJ, 516, 887. We use the trace hydrogen atmospheric temperatures.

[TVS:] Temperatures from IUE + optical data from Thejl P., Vennes S., Shipman H. L. 1991, ApJ, 370, 355.

[W:] Temperature from Liebert J. et al. 1986, ApJ, 309, 241, using Wesemael model atmospheres

[K:] Temperature from Liebert J. et al. 1986, ApJ, 309, 241, using Koester model atmospheres

[O:] Temperature from Liebert J. et al. 1986, ApJ, 309, 241, using optical data only

\section{Representative references for Table 5 (DOV and PNNV stars)}

1. PG 0122+200: Bond H.E., Grauer A. D. 1987, ApJ, 321, L123; Vauclair G. et al. 1995, A\&A, 299, 707; O'Brien M.S. et al. 1996, ApJ, 467, 397.

2. PG 1159-035: Winget D.E. et al. 1991, ApJ, 378, 326; Kawaler S. D., Bradley P. A. 1994, ApJ, 427, 415.

3. PG 1707+427: Bond H.E., Grauer A.D., Green R.F., Liebert J. 1984, ApJ, 279, 751; Fontaine G. et al. 1991, ApJ, 378, L49; Grauer A. D., Green R. F., Liebert J. 1992, 399, 686.

4. PG 2131+066: Bond H.E., Grauer A.D., Green R.F., Liebert J. 1984, ApJ, 279, 751; Kawaler S. D. et al. 1995, ApJ, 450, 350.

5. HS 2324+3944: Silvotti R. 1996, A\&A, 309, L23; Dreizler S. et al. 1996, A\&A, 309, 820; Handler G. et al. 1997, A\&A, 326, 692.

6. NGC 246: Ciardullo R., Bond H.E. 1996, AJ, 111, 2332. 
7. NGC 1501: Ciardullo R., Bond H.E. 1996, AJ, 111, 2332; Bond H.E. et al. 1996, AJ, 112, 2699.

8. NGC 2371-2: Bond H. E., Ciardullo R. 1993, White Dwarfs: Advances in Observations and Theory, ed. M. A. Barstow (NATO ASI ser.), Kluwer, Dordrecht, p. 491; Ciardullo R., Bond H. E. 1996, AJ, 111, 2332.

9. NGC 2867: Ciardullo R., Bond H. E. 1996, AJ, 111, 2332.

10. Lo-4: Longmore A. J. 1977, MNRAS, 178, 251; Bond H.E., Meakes M. G. 1990, AJ, 100, 788.

11. NGC 5189: Ciardullo R., Bond H.E. 1996, AJ, 111, 2332.

12. Sanduleak 3: Bond H. E., Ciardullo R. 1993, White Dwarfs: Advances in Observations and Theory, ed. M. A. Barstow (NATO ASI ser.), Kluwer, Dordrecht, p. 491.

13. K 1-16: Grauer A. D. Bond H.E. 1984, ApJ, 277, 211; Grauer A.D., Bond H. E., Green R.F., Liebert J. 1987, in The Second Conference on Faint Blue Stars, eds. A. 'G. D. Philip, D. S. Hayes \& J. W. Liebert, L. Davis Press, Schenectady, N.Y., p. 231.

14. NGC 6905: Bond H.E., Ciardullo R. 1993, in White Dwarfs: Advances in Observations and Theory, ed. M. A. Barstow (NATO ASI ser.), Kluwer, Dordrecht, p. 491; Ciardullo R., Bond H.E. 1996, AJ, 111, 2332.

15. RXJ 2117+3412: Watson T. K. 1992, IAU Circ., No. 5603; Vauclair G. et al. 1993, A\&A, 267, L35; Motch C., Werner K., Pakull M. W. 1993, A\&A, 268, 561.

\section{Representative references for Table 5 (Nonvariable stars)}

1. RXJ 0122.9-7521: Cowley A.P. et al. 1995, PASP, 107, 927; Bond H.E. 1996, priv. comm.; Werner K. et al. 1996, in Supersoft X-ray Sources, ed. J. Greiner, Lecture Notes in Physics, 472, Springer, Berlin, p. 131.

2. MCT 0130-1937: Demers S. et al. 1990, ApJ, 351, 271; Dreizler S., Heber U. 1998, A\&A, 334, 618.

3. HS 0444+0453: Dreizler S. 1995, in The 9th European Workshop on White Dwarf Stars, eds. D. Koester \& K. Werner, Lecture Notes in Physics, 443, Springer, Berlin, p. 160.

4. HS 0704+6153: Dreizler S. et al. 1994, A\&A, 286, 463; Dreizler S., Heber U. 1998, A\&A, 334, 618.

5. VV 47: Liebert J. et al. 1988, PASP, 100, 187; Dreizler S. 1995, in The 9th European Workshop on White Dwarf Stars, eds. D. 
Koester \& K. Werner, Lecture Notes in Physics, 443, Springer, Berlin, p. 160; Ciardullo R., Bond H. E. 1996, AJ, 111, 2332.

6. PG 1144+005: Grauer A.D. et al. 1987, ApJ, 323, 271; Werner K., Heber U. 1991 A\&A, 247, 746; Dreizler S. 1995, in The 9th European Workshop on White Dwarf Stars, eds. D. Koester \& K. Werner, Lecture Notes in Physics, 443, Springer, Berlin, p. 160.

7. PG 1151-029: Grauer A.D. et al. 1987, ApJ, 323, 271; Dreizler S. 1995, in The 9th European Workshop on White Dwarf Stars, eds. D. Koester \& K. Werner, Lecture Notes in Physics, 443, Springer, Berlin, p. 160.

8. PG 1424+535: Grauer A.D. et al. 1987, ApJ, 323, 271; Dreizler S., Heber U. 1998, A\&A, 334, 618.

9. H 1504+65: Nousek J. A. et al. 1986, ApJ, 309, 230; Grauer A.D. et al. 1987, ApJ, 323, 271; Barstow M. A., Tweedy R.W. 1990, MNRAS, 242, 484; Werner K. 1991, A\&A, 251, 147; Werner K. Wolff B. 1999, A\&A, 347, L9.

10. HS 1517+7403: Dreizler S. et al. 1994, A\&A, 286, 463; Dreizler S., Heber U. 1998, A\&A, 334, 618.

11. PG 1520+525: Grauer A. D. et al. 1987, ApJ, 323, 271; Dreizler S., Heber U. 1998, A\&A, 334, 618.

\section{Representative references for Table 6 (IBWD stars)}

1. CP Eri: Abbott T.M. C., Robinson E. L., Hill G. J., Haswell C. A. 1992, ApJ, 399, 680.

2. HZ 29: Solheim J.-E., Robinson E. L., Nather R.E., Kepler S. O. 1984, A\&A, 135, 1; Patterson J., Halpern J., Shambrook A. 1993, ApJ, 419, 803; Provencal J. L. et al. 1995, ApJ, 445, 927.

3. G 61-29: Nather R. E., Robinson E. L., Stover R. J. 1981, ApJ, 244, 269; Stover R. J. 1983, PASP, 95, 18; Marsh T.R., Wood J. H., Horne K., Lambert D. 1995, MNRAS, 274, 452; Marsh T. R. 1999, MNRAS, 304, 443.

4. V 803 Cen: Kepler S. O., Steiner J.E., Jablonski F. 1989, in White Dwarfs (IAU Colloq. 114), ed. G. Wegner, Springer, Berlin, p. 443; O'Donoghue D., Kilkenny D. 1989, MNRAS, 236, 319; Ulla A. M., Solheim J.-E. 1991, in The 7th European Workshop on White Dwarfs, eds. G. Vauclair \& E.M.Sion, Kluwer, Dordrecht, p. 441.

5. PG 1346+082: Wood M. A., Winget D. E., Nather R. E., Hessman F. V., Liebert J., Kurtz D. W., Wesemael F., Wegner G. 
1987, ApJ, 313, 757; Provencal J.L. et al. 1997, ApJ, 480, 383; Patterson J. et al. 1997, PASP, 109, 1100.

6. EC 1533-1403: O'Donoghue D., Kilkenny D., Chen A., Stobie R.S., Koen C., Warner B., Lawson W.A. 1994, MNRAS, 271, 910. 


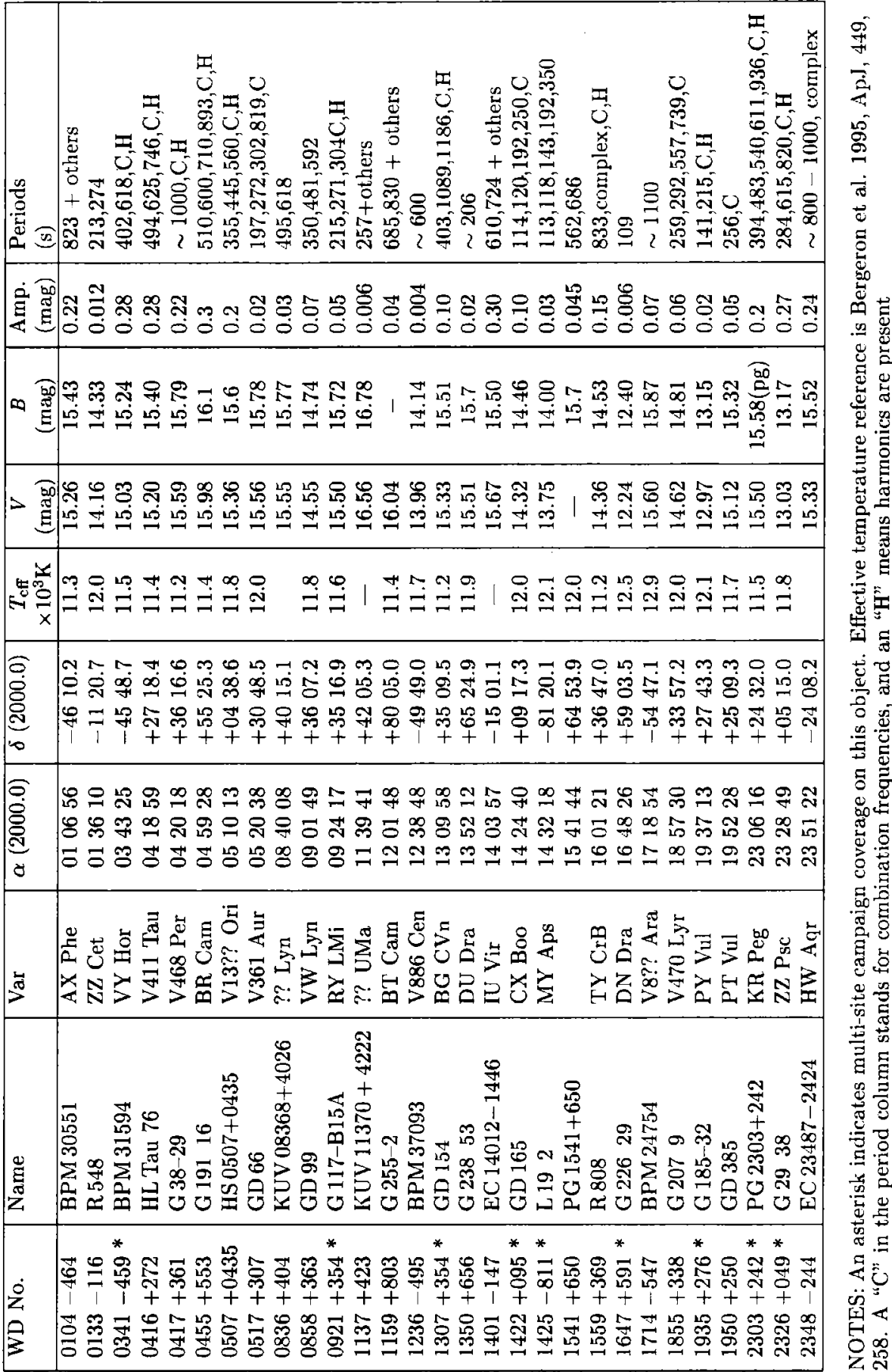




\begin{tabular}{|c|c|c|c|c|}
\hline $\mid \begin{array}{ll}\infty & 0 \\
0 & 0 \\
1 & 0 \\
0 & 0\end{array}$ & 我 & $\underset{\infty}{\infty} \underset{\infty}{\infty}$ & & $\begin{array}{lllll}\vec{N} & \sim & 0 & 0 & 0 \\
\infty & \infty & \infty & \infty & \stackrel{\infty}{N}\end{array}$ \\
\hline$\sum^{\infty}$ & 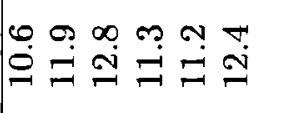 & 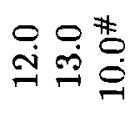 & & \\
\hline $\mid \begin{array}{ll}0 & 0 \\
\infty & E \\
3 & E\end{array}$ & $\stackrel{\infty}{\infty}$ & 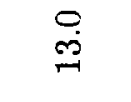 & $\stackrel{=}{=}$ & 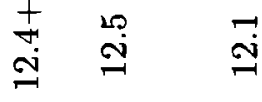 \\
\hline 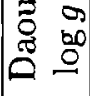 & $\stackrel{\infty}{N}$ & $\stackrel{\infty}{\infty}$ & $\underset{\infty}{\sim} \underset{\infty}{\infty} \stackrel{\infty}{\infty} \underset{\infty}{\infty}$ & $\underset{\infty}{\infty}$ \\
\hline 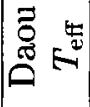 & $\begin{array}{l}\infty \\
\stackrel{\infty}{\sim}\end{array}$ & $\stackrel{\infty}{\stackrel{\infty}{\beth}}$ & 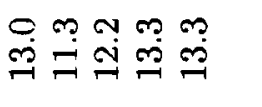 & $\stackrel{0}{\ddot{m}}$ \\
\hline Z & $\stackrel{\infty}{\rightleftharpoons}$ & $\underset{\nearrow}{\beth}$ & 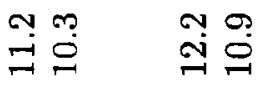 & 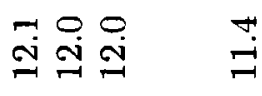 \\
\hline $\begin{array}{ll}0 \\
2 & 0 \\
0 & 0\end{array}$ & & $\stackrel{\infty}{\infty}$ & $\stackrel{\infty}{\infty} \stackrel{0}{\infty}$ & 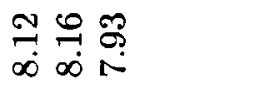 \\
\hline$\pi E^{\frac{\omega}{d}}$ & & ت゙ & 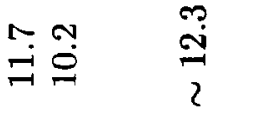 & 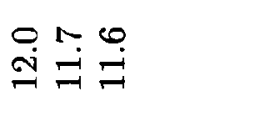 \\
\hline $\begin{array}{ll}\dot{0} & 0 \\
0 & 0 \\
\oplus & 0\end{array}$ & 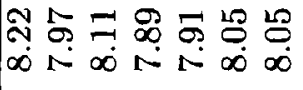 & $\stackrel{\infty}{\infty} \underset{\infty}{\infty} \stackrel{\Gamma}{\infty}$ & 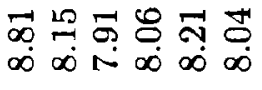 & 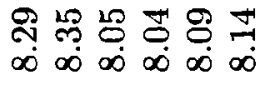 \\
\hline صृ & 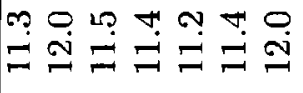 & 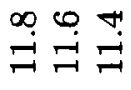 & 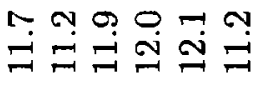 & 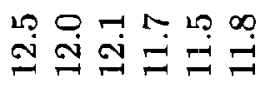 \\
\hline 胥 & 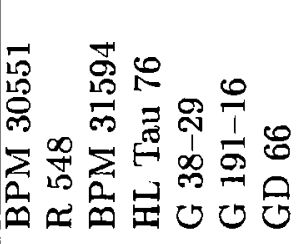 & 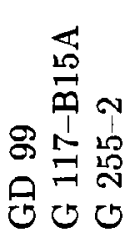 & 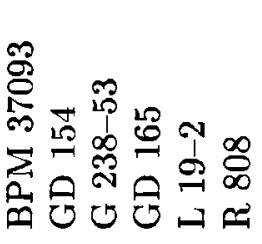 & 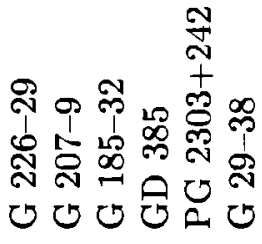 \\
\hline$\sum_{0}^{0}$ & 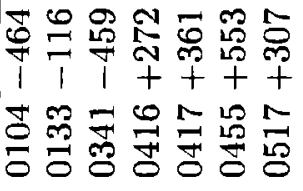 & 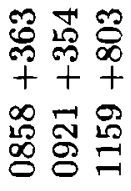 & 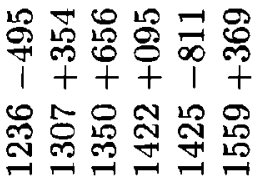 & 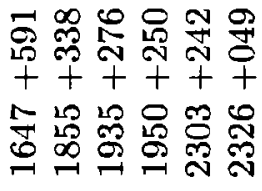 \\
\hline
\end{tabular}




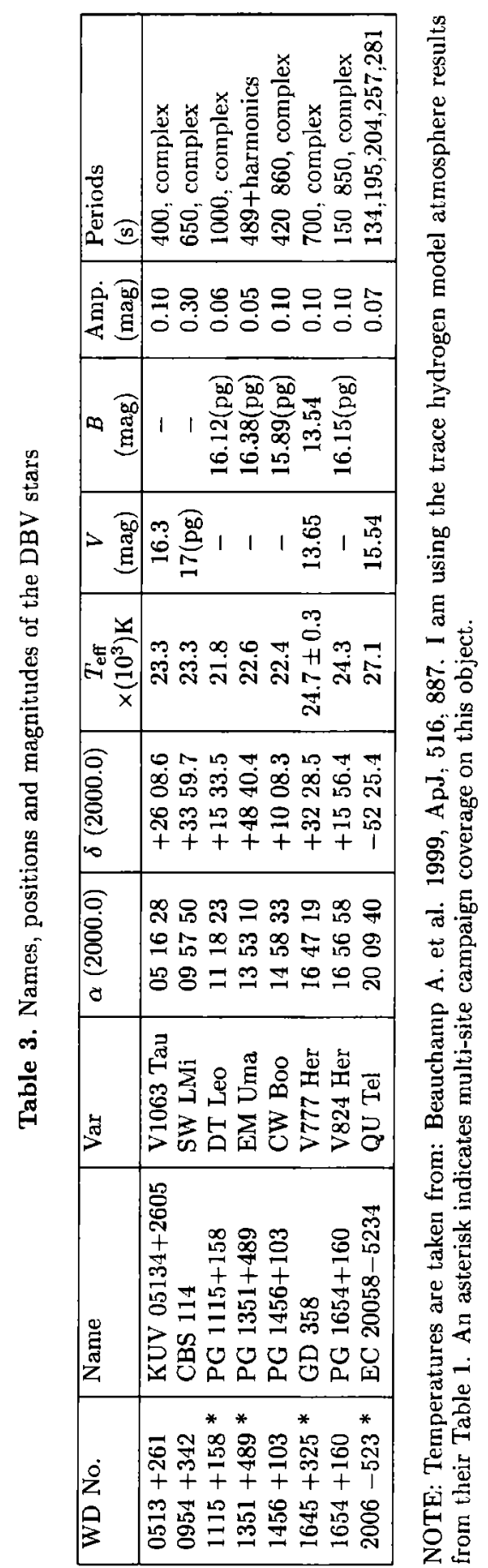




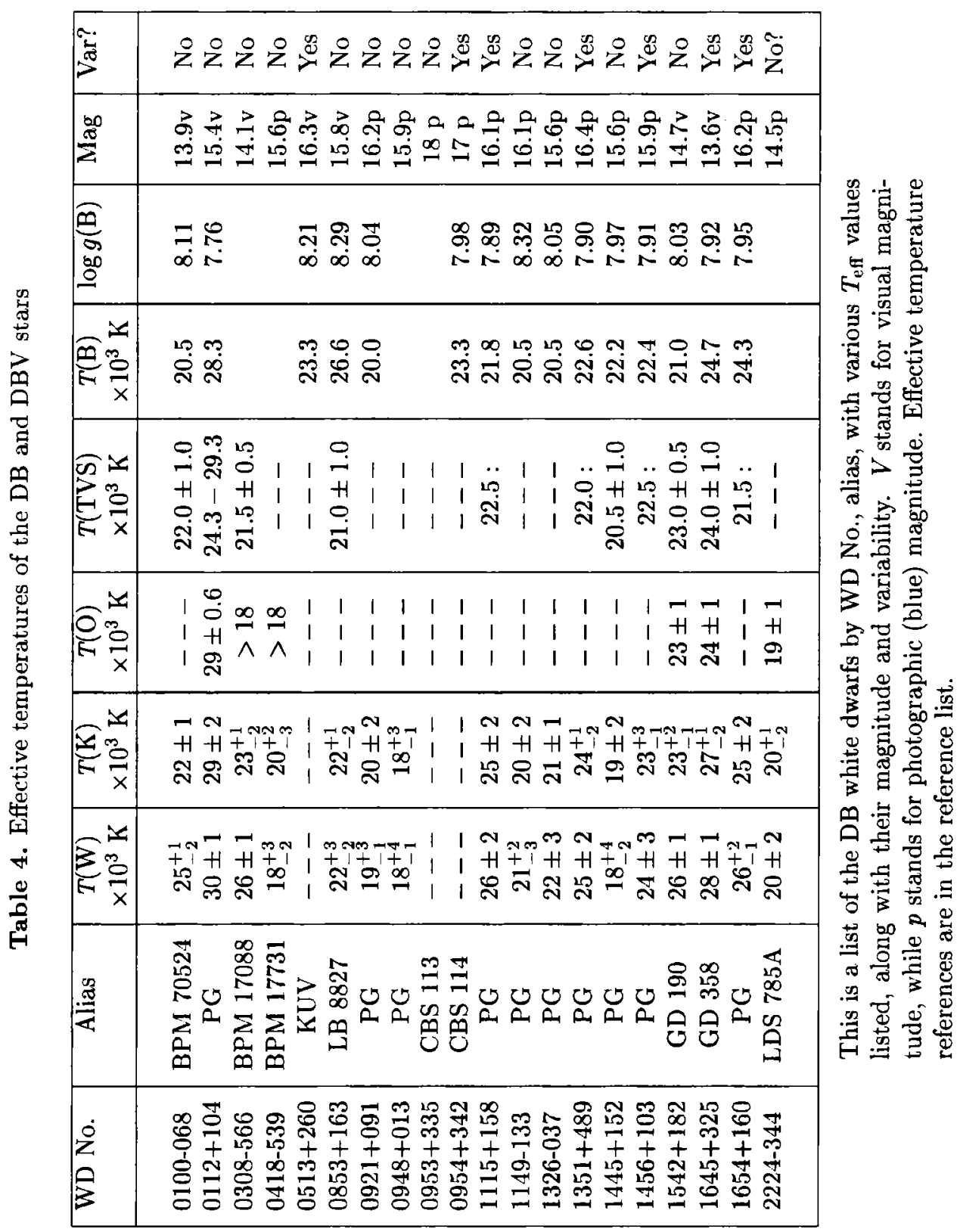




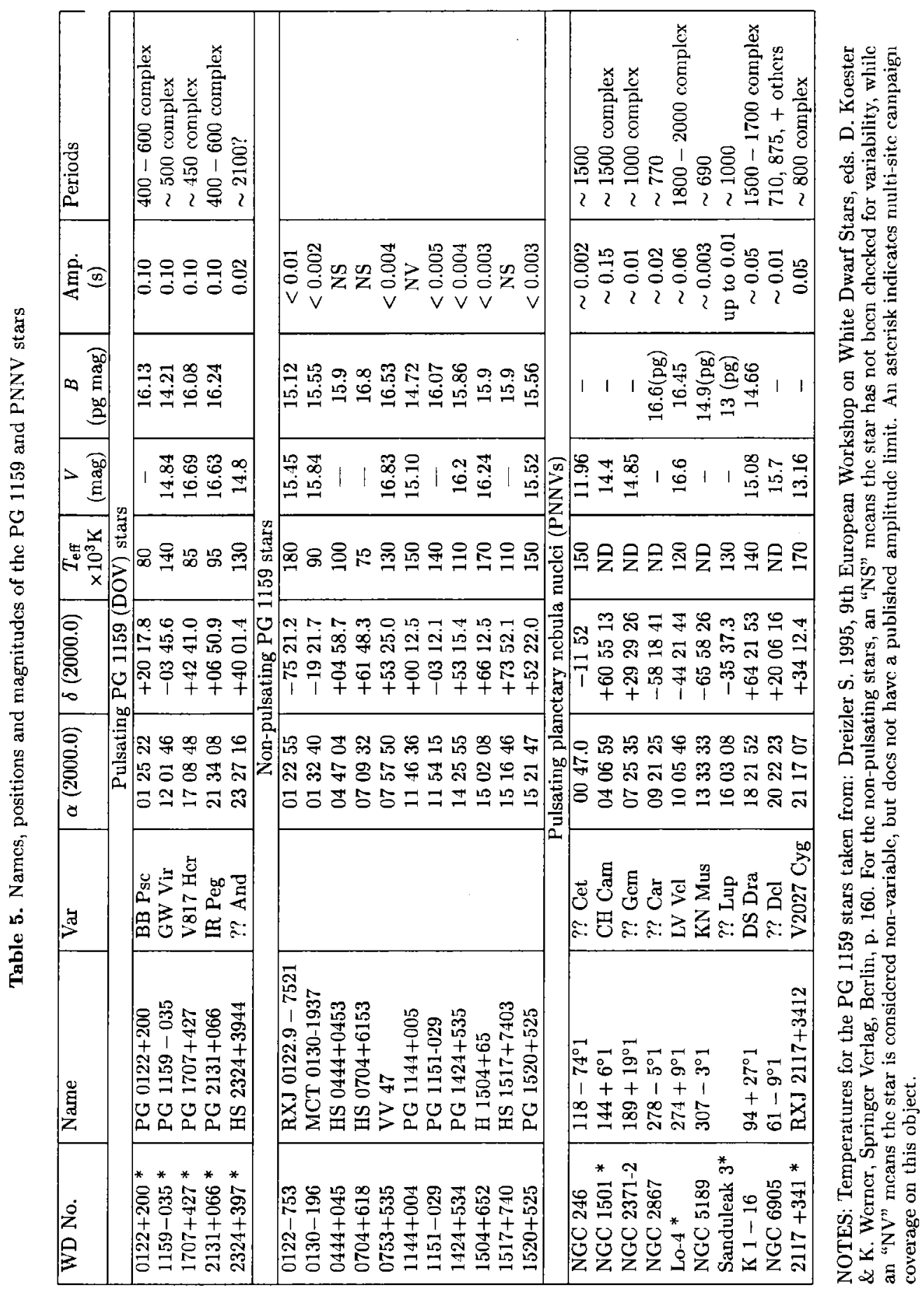




\begin{tabular}{|c|c|c|}
\hline & $\stackrel{\vec{D}}{0} \widetilde{0}$ & 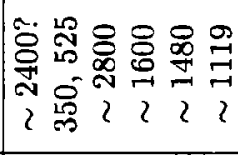 \\
\hline 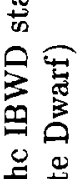 & 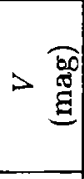 & 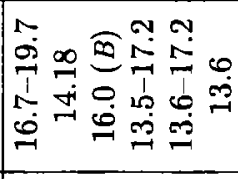 \\
\hline $\begin{array}{ll}0 \\
0\end{array}$ & 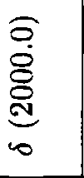 & 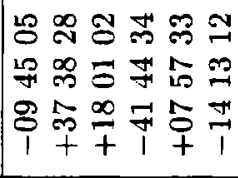 \\
\hline $\begin{array}{ll}0 \\
0 \\
0\end{array}$ & 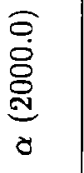 & 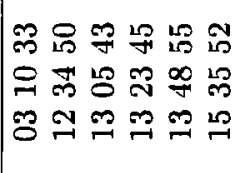 \\
\hline ? & $\stackrel{\mathscr{G}}{\stackrel{\Xi}{二}}$ & 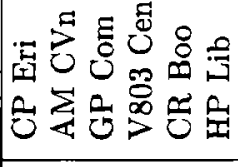 \\
\hline$\stackrel{\theta}{\theta}$ & 宽 & 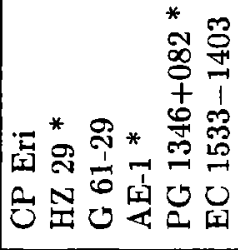 \\
\hline
\end{tabular}

